Forum 2021 · 36:299-304

https://doi.org/10.1007/s12312-021-00963-8

Online publiziert: 12. August 2021

๑) Springer Medizin Verlag GmbH, ein Teil von Springer Nature 2021

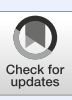

\section{Autor}
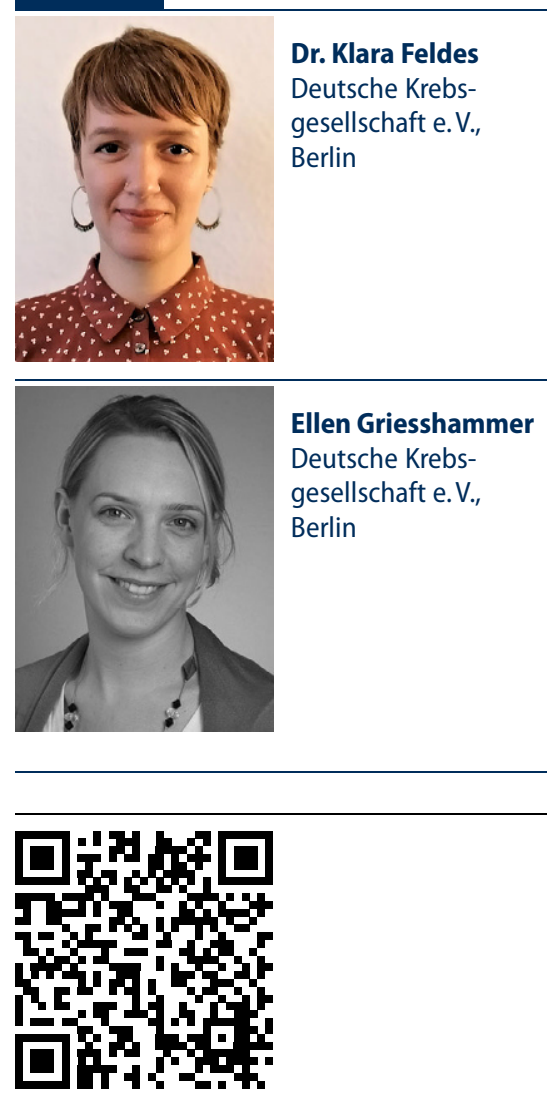

QR-Code scannen \& Beitrag online lesen

\title{
Europas Kampf gegen Krebs: Europe's Beating Cancer Plan
}

\section{Let's strive for more}

\author{
Klara Feldes ${ }^{1}$ Ellen Griesshammer ${ }^{2}$ \\ 'Bereich Wissensmanagement/Infonetz Krebs, Deutsche Krebsgesellschaft e. V., Berlin, Deutschland \\ ${ }^{2}$ Bereich Zertifizierung, Deutsche Krebsgesellschaft e.V., Berlin, Deutschland
}

\section{Zusammenfassung}

Auf Ebene der Europäischen Union steht derzeit im Bereich Gesundheit die Krebsbekämpfung ganz oben auf der Prioritätenliste. In diesem Zusammenhang legte die Europäische Kommission im Februar 2021 den Europäischen Krebsplan (Europe's Beating Cancer Plan, EBCP) vor. Dieser Beitrag gibt Einblick in die Hintergründe, den Aufbau und die Implementierung des EBCP. Ausgewählte Maßnahmen des EBCP anhand der 4 Säulen Prävention, Früherkennung, Diagnose und Behandlung sowie Verbesserung der Lebensqualität von Krebspatient(inn)en und -überlebenden werden beleuchtet. Auch wenn vieles noch am Anfang steht, wirft der Beitrag zudem einen Blick auf die ersten Steuerungs- und Monitoringinitiativen, die die EU zur Umsetzung des Plans ergriffen hat. Dabei steht auch die fachlich übergreifende Zusammenarbeit zwischen Forschung und Versorgung - wie sie ihm Krebsplan angelegt ist und auch in Deutschland zunehmend stattfindet - im Fokus.

\section{Schlüsselwörter}

Gesundheitspolitik · Europapolitik · Krebsfrüherkennung · Lebensqualität · Versorgungsqualität

Der Europäische Krebsplan (Europe's Beating Cancer Plan. EBCP) ist die FlaggschiffInitiative der Europäischen Kommission zur Unterstützung der Krebsbekämpfungsmaßnahmen in den europäischen Mitgliedsstaaten.

Dabei sind die Heterogenität der nationalen Gesundheitssysteme und die damit verbundenen strukturellen und finanziellen Unterschiede immer wieder eine Herausforderung. Hinzu kommen aktuell die Auswirkungen der COVID-19-Pandemie auf die Krebsversorgung, z.B. verzögerte Diagnosen oder erschwerter Zugang zu Medikamenten [1].

Trotz Pandemie werden die europäischen Mitgliedsstaaten durch die vielfältigen Aufgaben, die onkologische Erkrankungen mit sich bringen, vereint. Die komplexen Krankheitsverläufe, vielfältigen Therapieansätze und rasanten wissenschaftlichen Fortschritte sowie die Prognose, dass die onkologischen Neuerkrankungen ohne strukturelle Verän- derungen bis 2035 um fast $25 \%$ in der Europäischen Union (EU) ansteigen werden, machen eine enge Zusammenarbeit auf europäischer Ebene unumgänglich [2]. Das erklärte Ziel lautet daher, gemeinsam in allen Mitgliedsstaaten die Entstehung von Krebs zu verhindern und an Krebs erkrankten Menschen möglichst die gleichen hohen Standards anbieten zu können.

Der letzte europäische Aktionsplan gegen Krebs stammt aus den frühen 1990er Jahren [1]. Mit dem neuen Europäischen Krebsplan ist nun der richtige Zeitpunkt gekommen, um eine aktualisierte gemeinsame europäische Strategie für die Verbesserung der onkologischen Versorgung zu etablieren.

\section{Aufbau und Umsetzung des Europäischen Krebsplans}

Durch einen innovativen Entwicklungsprozess wurde der Plan nach einer breitge- 
fächerten Konsultationsphase mit Interessenvertreter(inne)n und EU-Bürger(inne)n innerhalb von nur 12 Monaten erstellt.

Dabei wurden 4 Säulen identifiziert, aus denen sich 10 Leitinitiativen und eine Vielzahl an unterstützenden Maßnahmen ableiten. Die 4 Säulen des EBCP, 1) Prävention, 2) Früherkennung, 3) Diagnose und Behandlung sowie 4) Verbesserung der Lebensqualität von Krebspatient(inn)en und -überlebenden, werden von den Querschnittsthemen Forschung und Innovation, digitale und personalisierte Medizin sowie Abbau von Ungleichheiten innerhalb der europäischen Mitgliedsstaaten flankiert. Ein besonderes Augenmerk wird auf Kinder gelegt, um einen Zugang zu schneller und optimaler Erkennung, Diagnose, Behandlung und Pflege sicherzustellen.

Nicht der Tumor soll im Fokus stehen, sondern der Mensch. Auch die nichtsichtbaren psychischen und psychosozialen Folgen der Erkrankung, Stigmatisierung und Diskriminierung, sollen verhindert und die höchstmögliche Lebensqualität soll erreicht werden [3].

Der insgesamt mit 4Mrd. EUR ausgestattete Europäische Krebsplan wurde am 3. Februar 2021, dem Vorabend des Weltkrebstages, veröffentlicht.

Die Mittel stammen größtenteils aus dem künftigen EU4Health-Programm $(1,25 \mathrm{Mrd}$. EUR), verantwortet von der Generaldirektion Gesundheit (DG Santé), und dem neuen Förderprogramm Horizon Europe (2 Mrd. EUR) unter der Leitung der Generaldirektion Forschung und Innovation (DG RTD). Ziel dieses gemeinsamen Finanzierungsansatzes ist es, bürokratische Silos zu überwinden, die Konzentration auf gemeinsame Ziele zu fördern und die schnelle Identifizierung von Synergien über die Grenzen verschiedener thematischer Generaldirektionen hinweg zu ermöglichen [4].

\section{Ausgewählte inhaltliche Schwerpunktthemen}

\section{Präventionsmaßnahmen}

Europas Plan gegen den Krebs beschäftigt sich ausführlich mit dem Themenbereich Prävention und nimmt dabei verschiedene Handlungsbereiche in den Blick. Als themenübergreifende Maßnahme gehört dazu die Aktualisierung des bestehenden Europäischen Kodex zur Krebsbekämpfung. Dieser umfasst 12 Aspekte (u.a. Nichtrauchen, Screeningteilnahme) und zielt auf die individuelle Umsetzung eines gesunden Lebensstils. Die Aktualisierung soll neuer wissenschaftlicher Evidenz sowie neuen Erkenntnissen im Bereich der Risikokommunikation Rechnung tragen. Welche Änderungen sich daraus konkret ergeben, ist jedoch noch unklar. Bis 2025 sollen $80 \%$ der europäischen Bevölkerung für den Kodex sensibilisiert sein, u.a. mithilfe einer EU-App, die über Krebsprävention aufklärt, und durch ein Projekt zur Stärkung von Krebspräventions- und -versorgungsprogrammen mit Schwerpunkt auf vulnerablen Gruppen.

Einer der Teilaspekte der anvisierten Präventionsmaßnahmen zielt auf die Eindämmung des Tabakkonsums: Der dafür vorhandene EU-Rahmen soll angepasst und rigoroser umgesetzt werden. Außerdem sind strengere Vorschriften zu neuartigen Produkten vorgesehen. Seit Jahren hinkt Deutschland im EU-Vergleich bei der Eindämmung des Tabakkonsums hinterher. Auch in der 2019 zuletzt erstellten EU-Tabakkontrollskala belegt Deutschland den letzten Platz [5]. Am 1. Januar 2021 ist jedoch das neue Tabakwerbeverbot in Kraft getreten, das nun stufenweise umgesetzt wird [6]. Auch das derzeit debattierte Gesetz zur Modernisierung des Tabaksteuerrecht nimmt sich der Aufgabe der Verringerung des Tabakkonsums an [7]. Die Deutsche Krebsgesellschaft (DKG) begrüßt das geplante Tabaksteuermodernisierungsgesetz, „denn Tabaksteuererhöhungen zählen, wenn sie mit spürbaren Preiserhöhungen einhergehen, zu den wirksamsten Mitteln der Tabakprävention" [8]. Somit wurden erste Schritte eingeleitet, um das Ziel des EBCP, den Tabakkonsum bis zum Jahre 2040 so weit zu senken, dass weniger als $5 \%$ der Bevölkerung Tabak konsumieren, zu erreichen.

Eine der beschriebenen Maßnahmen im Bereich Gesundheitsförderung durch gesündere Ernährung und Bewegung ist eine mögliche verpflichtende Nährwertkennzeichnung auf der Vorderseite von Lebensmittelverpackungen. In Deutschland gibt es seit 2020 den sog. Nutri-Score, die Lebensmittelampel, bisher jedoch nur auf freiwilliger Basis. Eine EU-weite Regelung könnte hier zu einer erhöhten Verbindlichkeit führen.

Auch das umstrittene Thema der Regulierung von Solariengeräten wird kurz erwähnt, der Krebsplan kündigt hier jedoch keinen festen Fahrplan für Änderungen an. Geeignete Maßnahmen sollen hierbei von der Kommission ausgelotet werden. Das Problem der Nichtzuständigkeit der DG Santé - derzeit liegt die Regulierung von Solariengeräten im Arbeitsbereich der Generaldirektion Binnenmarkt, Industrie, Unternehmertum und KMU (DG GROW) scheint hier weiterhin konkretere Pläne zu verhindern.

\section{Impfen gegen Krebs}

Neben weiteren präventiven Komponenten des Krebsplans steht auch die Prävention von Krebserkrankungen, die durch Infektionen verursacht werden, im Fokus. Dieser wichtigen Aufgabe wird sich die 3. Nationale Krebspräventionswoche vom 13.-17. September 2021 mit dem Thema „Impfen gegen Krebs" widmen. Die Krebspräventionswoche wird gemeinsam von der Deutschen Krebshilfe, dem Deutschen Krebsforschungszentrum und der DKG durchgeführt.

Laut Europäischem Krebsplan soll insbesondere die Impfkampagne für Mädchen und Jungen gegen humane $\mathrm{Pa}$ pillomaviren (HPV) ausgeweitet werden, um Gebärmutterhalskrebs und andere Krebserkrankungen einzudämmen. Die Leitinitiative 3 zielt darauf $a b$, dass bis zum Jahr 2030 mindestens $90 \%$ aller Mädchen in der EU geimpft werden und die Impfquote bei den Jungen deutlich erhöht ist. Laut Zahlen des Robert Koch-Instituts [9] betrug im Sommer 2020 die Quote der HPV-Impfungen in Deutschland bei Mädchen $43 \%$ - mit großen regionalen Unterschieden (34\% in Baden-Württemberg, $63 \%$ in Sachsen-Anhalt). Jungen werden in Deutschland seit 2018 gegen HPV geimpft. Deutlich höhere Impfquoten haben hier bisher Länder mit bestehenden Schulimpfprogrammen. Auch die Ständige Impfkommission wies in ihrer Empfehlung zur HPV-Impfung für Jungen (2018) darauf hin, dass die Implementierung deutschlandweiter Schulimpfprogramme erwogen werden sollte, um möglichst 
Hier steht eine Anzeige.

黑 Springer 
hohe HPV-Impfquoten zu erzielen [10]. In diesem Bereich wird somit in den nächsten Jahren deutlicher Handlungsbedarf bestehen. Eine Frage lässt der Krebsplan dabei jedoch offen: Weltweit gibt es zurzeit eine Knappheit des HPV-Impfstoffs. Mindestens noch bis in das Jahr 2024 wird dieses Problem fortbestehen - wie wirkt sich das auf das Erreichen der Zielstellung aus [11]?

Neben der Stärkung der HPV-Impfung soll laut Krebsplan auch der Zugang zur Hepatitis-B-Impfung für die Prävention von Leber- und Magenkrebs verbessert werden. In Deutschland lag die Quote der Hepatitis-B-Impfung bei Kindern zum Zeitpunkt der Schuleingangsuntersuchung im Jahr 2017 bei $86,9 \%$ [12].

\section{Verbesserte Früherkennung}

Eine weitere Säule des Krebsplans ist die verbesserte Früherkennung, insbesondere in den Bereichen Brust-, Gebärmutterhalsund Darmkrebs. Laut Krebsplan bestanden im Jahr 2020 populationsspezifische Vorsorgeprogramme für Brustkrebs in 25 , für Gebärmutterhalskrebs in 22 und für Darmkrebs in 20 Mitgliedsstaaten - allerdings mit sehr heterogenen Erfolgen. So schwankt die Zielgruppenabdeckung im Vergleich der EU-Länder beispielsweise bei der Brustkrebsvorsorge zwischen $6 \%$ und $90 \%$. Hier wird dringender Handlungsbedarf im Angleich der Versorgung in den verschiedenen Mitgliedsstaaten gesehen.

Im Rahmen der Leitinitiative 4 sollen die Mitgliedsstaaten dabei unterstützt werden, bis 2025 90\% der infrage kommenden EU-Bürger(inne)n Vorsorgeuntersuchungen in diesen drei Bereichen anzubieten. In Deutschland gibt es seit 2009 das flächendeckend organisierte Mammographiescreeningprogramm. Im Rahmen des Nationalen Krebsplans wurde im Jahr 2019 zudem auch ein organisiertes Darmkrebsscreening eingeführt. Im Januar 2020 folgte dann das Gebärmutterhalsscreening. Seit diesem Zeitpunkt werden anspruchsberechtigte Frauen von ihren Krankenkassen angeschrieben und zu einem Screening eingeladen. In diesem Teilbereich des Krebsplans ist die Implementierung in Deutschland somit bereits vorangeschritten.
Verbesserung der Versorgungsstandards

Der Strategieplan betont im Themenbereich 5 „Gewährleistung hoher Standards bei der Versorgung von Krebskranken“ unter der Leitinitiative 5 u. a. die Bildung eines EU-Netzwerkes bestehend aus nationalen onkologischen Spitzenzentren (Comprehensive Cancer Centres) sowie deren Qualitätssicherung. In einem ersten Schritt soll ein Mapping der onkologischen Spitzenzentren in den Mitgliedsstaaten dazu beitragen, die verschiedenen in der EU verfügbaren Fähigkeiten und Fachkompetenzen zu erfassen und diese in einem zweiten Schritt miteinander zu vernetzen.

Die Relevanz des DKG-Zertifizierungssystems auf europäischer Ebene wird in dieser Leitinitiative mehr als deutlich. Wie genau die langjährige Erfahrung der DKG in die Gestaltung der onkologischen Versorgung für Europa einfließen kann, ist noch unklar. Zurzeit bestehen noch einige Unschärfen bei den Begrifflichkeiten und Definitionen, z. B.: Welche Anforderungen muss ein nationales onkologisches Spitzenzentrum erfüllen, wie findet das Auswahlverfahren statt? Gibt es eine Benennung durch eine externe Zertifizierungsstelle? Ebenfalls ist unklar, ob die Forderung nach Zugang für $90 \%$ der „berechtigten Patienten" zu nationalen onkologischen Spitzenzentren bis 2030 ein sinnvolles und realisierbares Ziel ist. Derzeit gilt es noch, den Schlussbericht des Cancer Mission Board abzuwarten. Die „missions" wurden im Rahmen von Horizon Europe ins Leben gerufen und sollen verschiedene Stakeholder, Fachbereiche und Innovationen miteinander verknüpfen und zusammenbringen. Im Bereich Onkologie ist ein zentrales Anliegen der Cancer Mission, bei neuen Forschungsergebnissen einen starken Versorgungsbezug und eine Umsetzbarkeit der erarbeiteten Empfehlungen sicherzustellen. Im Juni 2020 hat das Cancer Mission Board seinen ersten Report mit dem Titel "Conquering cancer, mission possible" veröffentlicht. Der Bericht enthält 13 Empfehlungen, u.a. den Aufbau umfassender onkologischer Infrastrukturen (Comprehensive Cancer Infrastructure) in und zwischen allen EU-Mitgliedsstaaten, um die Qualität von Forschung und Versorgung zu verbessern („Cancer Mission interim report" [13]). Es wird davon ausgegangen, dass die im Europäischen Krebsplan beschriebenen onkologischen Spitzenzentren Teil der Comprehensive Cancer Infrastructures sind. Der Schlussbericht der Cancer Mission sowie das Arbeitsprogramm, in dem die erforderlichen Maßnahmen und Strategien zur Erreichung der Ziele beschrieben werden, wird Ende 2021 erwartet.

\section{Rehabilitation und Survivorship}

Neben den Zielen, Krebs zu verhindern und eine qualitativ hochwertige Versorgung sicherzustellen, widmet sich der EBCP auch dem Ziel, nach Krebs und mit Krebs als chronischer Erkrankung gut und lange zu leben. Der Krebsplan erkennt an, dass es immer mehr Krebsüberlebende gibt, die in den Blick genommen werden müssen. Dabei soll der Fokus nicht mehr nur auf der Frage, wie lange Patienten überleben, liegen, sondern auch auf der Frage, wie gut sie dies tun. Die Leitinitiative 8 des EBCP beinhaltet u.a. die Einführung eines freiwilligen elektronischen Passes für Krebsüberlebende, der einer besseren Kommunikation und Koordinierung der medizinischen Nachsorge dienen soll. Um auch die finanziellen Folgen einer Krebserkrankung besser abzufedern, soll Betroffenen die Rückkehr in das Berufsleben erleichtert werden. Auch Umschulungs- und Weiterbildungsprogramme werden unterstützt.

Das Thema Cancer Survivors hat nicht nur auf EU-Ebene in den letzten Jahren zunehmend an Bedeutung gewonnen. Auch die Deutsche Krebsstiftung organisiert jährlich den German Cancer Survivors Day und in diesem Jahr erstmalig die German Cancer Survivors Week.

Genauso wie es wichtig ist, Krebsüberlebenden den Raum für Vernetzung und Austausch anzubieten, wird zunehmend auch über das "Recht auf Vergessen“ [14] diskutiert. Dabei fordern Krebsüberlebende, nicht langfristig aufgrund ihrer überstandenen Krankheit benachteiligt zu werden. Dies betrifft u. a. das Abschließen von Versicherungen, Kreditvergaben oder auch Verbeamtungen, von denen Krebsüberlebende häufig ausgeschlossen sind oder nur zu sehr hohen Prämien aufgenommen werden. Diesem Diskurs trägt der EUKrebsplan insofern Rechnung, als dass der 
Plan vorsieht, dass die Kommission mit Unternehmen in einen Dialog tritt, um Diskriminierung von Krebsüberlebenden bei Finanzprodukten (insbesondere Kredit- und Versicherungsleistungen) entgegenzutreten.

\section{Steuerungen und Monitoring}

Zur Umsetzung der benannten Ziele setzt die EU auf die Erstellung einer Roadmap, die die Implementierung des Plans mithilfe zu definierender Indikatoren überwachen soll. Für die Erstellung hat die Kommission eine Lenkungsgruppe zur Umsetzung des EU-Krebsplans eingerichtet. Die Gruppe soll eng mit dem Europäischen Parlament, den Mitgliedsstaaten, dem Horizon Europe Krebsmissionsrat und der Stakeholder Contact Group zusammenarbeiten.

Eine zentrale Rolle für ein abgestimmtes und koordiniertes Vorgehen der beiden Generaldirektionen DG Santé und DG RTD soll das neue Knowledge Centre on Cancer, übernehmen, das an der gemeinsamen Forschungsstelle der EU (Joint Research (entre) angesiedelt ist.

Als innovative Maßnahme wurde eine Stakeholder Contact Group als weiteres Beratungs- und Konsultationsgremium initiiert. Die Einbindung der Stakeholder soll zum Ownership des Europäischen Krebsplans beitragen und die Arbeit der Lenkungsgruppe ergänzen. Bereits über 300 Interessenten haben sich auf der EUPlattform für Gesundheitspolitik (Health Policy Platform) registriert.

Die Interessen und Prioritäten des Europaparlaments und somit der europäischen Bürger(innen) werden durch einen Sonderausschuss zur Krebsbekämpfung (BECA) in der Lenkungsgruppe vertreten. Dieser Ausschuss besteht aus Mitgliedern des Europäischen Parlaments; zu seinen Aufgaben gehört es, Vorschläge der Generaldirektionen DG Santé und DG RTD zu kommentieren und Empfehlungen abzugeben.

Die Lenkungsgruppe wird beraten durch die Subgroup on Cancer, eine Untergruppe der Lenkungsgruppe für Gesundheitsförderung, Krankheitsprävention und Management von nichtübertragbaren Krankheiten innerhalb des DG Santé. In diesem Gremium werden die Mitgliedsstaaten über das geplante Vorgehen bei der Umsetzung des Krebsplans informiert und die erarbeiteten Vorschläge der Kommission kommentiert.

\section{Ausblick und Zusammenspiel europäischer und nationaler Aktivitäten}

Der Europäische Krebsplan ist angesichts seiner Vielzahl an Maßnahmen und der Verzahnung verschiedener Zuständigkeitsbereiche ein ambitioniertes und innovatives Projekt der Europäischen Kommission. Wie dieser Beitrag verdeutlicht, befinden sich die Koordinierungsund Governance-Strukturen im Aufbau und Implementierungsstrategien müssen erst noch entwickelt werden. In den Mitgliedsstaaten wird die Finanzierung und Umsetzung des EBCP insbesondere durch Ausschreibungen der EU4Health- und Horizon-Europe-Programme erfolgen.

Trotz des umfangreichen Programms darf nicht vergessen werden, dass die Aktivitäten der EU rein ergänzend zur nationalen Gesundheitspolitik stattfinden und ausschließlich eine koordinierende Funktion haben. Jedoch besteht in den Mitgliedsstaaten ein großes Interesse, sich an den Themenbereichen des ECBP auszurichten.

Mit Blick auf Deutschland ist es noch zu früh, um Aussagen zur Implementierung des EBCP auf nationaler Ebene zu machen. Derzeit erfolgen in allen Ministerien, die sich thematisch mit onkologischen Themen beschäftigen, eine Bestandsaufnahme und Abfrage, welche Themen des EBCP prioritär behandelt werden sollen. Dies geschieht - ganz wie im EBCP verankert - im Sinne eines „Health-in-All-Policies-Ansatzes". Dieser sieht vor, dass Gesundheitsaspekte in allen Bereichen der Politik mitgedacht werden.

Klar ist jedoch: Der Nationale Krebsplan, mit Fokus auf der Weiterentwicklung der Krebsversorgung, sowie die Nationale Dekade gegen Krebs, als Verbindung von Forschung (Bundesministerium für Bildung und Forschung, BMBF) und Versorgung (Bundesministerium für Gesundheit, BMG), werden sich der neuen Prioritäten annehmen und enger fachlich übergreifend zusammenarbeiten. Die Parallele zwischen der Vision der Nationalen Dekade gegen Krebs mit Beteiligung des BMG und des BMBF und der Vision des Europäischen
Krebsplan unter Beteiligung der DG Santé und der DG RTD, lässt hoffen, dass sich Versorgung und Behandlung im Sinne eines Ansatzes "from bench to bedside and back" (sinngemäß: vom Labor zum Krankenbett und zurück) bewährt und die Verzahnung von Versorgung und Forschung im Sinne einer Wissen generierenden onkologischen Versorgung vorantreibt [15].

Korrespondenzadresse

\section{Dr. Klara Feldes}

Bereich Wissensmanagement/Infonetz Krebs, Deutsche Krebsgesellschaft e.V.

Kuno-Fischer-Str. 8, 14057 Berlin, Deutschland feldes@krebsgesellschaft.de

\section{Einhaltung ethischer Richtlinien}

Interessenkonflikt. K. Feldes und E. Griesshammer geben an, dass kein Interessenkonflikt besteht.

Für diesen Beitrag wurden von den Autoren keine Studien an Menschen oder Tieren durchgeführt. Für die aufgeführten Studien gelten die jeweils dort angegebenen ethischen Richtlinien.

\section{Literatur}

1. Europäische Kommission Press release-europe's beating cancer plan: a new EU approach to prevention, treatment and care. https://ec.europa. eu/commission/presscorner/detail/en/ip_21 342 (Erstellt: 3. Febr. 2021). Zugegriffen: 4. Juni 2021

2. International Agency for Research on Cancer (2020up) Cancer Tomorrow-A tool that predicts the future cancer incidence and mortality burden worldwide from the current estimates in 2020 up until 2040. https://gco.iarc.fr/tomorrow/en. Zugegriffen:4. Juni 2021

3. Deutsche Sozialversicherung Europavertretung (2020) Themenletter ed* Nr.01/2020: Ein neuereuropäischer Krebsplan kommt. https://dsv-europa. de/de/themenletter/ed-nr.-01-2020/kapitel-2. html. Zugegriffen: 7. Juni 2021

4. Wallace N, Hudson RL New-look EU research department aims to overcome bureaucratic silos", Sci. Bus., Apr. 2019. https://sciencebusiness.net/ news/new-look-eu-research-department-aimsovercome-bureaucratic-silos. Zugegriffen: 4. Juni 2021

5. Joossens L, Feliu A, Fernandez E (2020) "The tobacco control scale in Europe 2019". A report of the association of European cancer leagues. https://www.tobaccocontrolscale.org/TCS2019. pdf.Zugegriffen: 14. Mai 2021

6. Bundestag (2021) Zweites Gesetz zur Änderung des Tabakerzeugnisgesetzes. https://www.bgbl.de/xaver/bgbl/start.xav? startbk=Bundesanzeiger_BGBI\&start $=\% 2 \mathrm{~F} \% 2 \mathrm{~F}$ \%2A\%5B\%40attr_id=\%27bgbl120s2229.pdf $\% 27 \% 5 \mathrm{D \#}$ _bgbl_\%2F\%2F*\%5B\%40attr id\%3D\%27bgbl120s2229.pdf\%27\%5D 1620986242563.Zugegriffen: 14. Mai 2021 
7. Entwurf eines Gesetzes zur Modernisierung des Tabaksteuerrechts (Tabaksteuermodernisierungsgesetz - TabStMoG). https://dip21.bundestag.de/ dip21/btd/19/286/1928655.pdf

8. Deutsche Krebsgesellschaft Pressemitteilung: Krebsexpert*innen befürworten geplantes Tabaksteuermodernisierungsgesetz. https://www.krebsgesellschaft.de/deutschekrebsgesellschaft-wtrl/willkommen/presse/ pressemitteilungen-2021/krebsexpert-innenbefuerworten-geplantes-tabaksteuermo.html (Erstellt: 17. Mai 2021).Zugegriffen:4. Juni 2021

9. Robert Koch Institut Epidemiologisches Bulletin 32/33 2020. https://www.rki.de/DE/Content/ Infekt/EpidBull/Archiv/2020/Ausgaben/32-33 20.pdf?_blob=publicationFile (Erstellt: 6 . Aug 2020). Zugegriffen: 14. Mai 2021

10. Robert Koch Institut Epidemiologisches Bulletin 26/2018. https://www.rki.de/DE/Content/Infekt/ EpidBull/Archiv/2018/Ausgaben/26_18.pdf? blob=publicationFile (Erstellt: 28. Juni 2018). Zugegriffen: 14. Mai 2021

11. WHO (2020) Global market study_HPV. https:// www.who.int/immunization/programmes_ systems/procurement/mi4a/platform/module2/ HPV_Global_Market_Study_Public_SummaryNov2020.pdf?ua=1.Zugegriffen: 14. Mai 2021

12. Robert Koch Institut EpidemiologischesBulletin 18/2019. https://www.rki.de/DE/Content/Infekt/ EpidBull/Archiv/2019/Ausgaben/18_19.pdf? blob=publicationFile (Erstellt:2. Mai 2019)

13. Europäische Kommission (2020) „Proposed Mission Conquering cancer: mission possible" https://op.europa.eu/en/publicationdetail/-/publication/b389aad3-fd56-11eab44f-01aa75ed71a1/https://op.europa.eu/en/ publication-detail/-/publication/b389aad3fd56-11ea-b44f-01aa75ed71a1/. Zugegriffen am: 28. Juli 2021

14. European Cancer Patient Coalition (2021) 14 million reasons to discuss life after cancer: Implementing the right to be forgotten across Europe. https://ecpc.org/wp-content/uploads/2021/03/ Right-to-be-Forgotten_Event-Report-1.pdf

15. Deutsche Krebsgesellschaft (2019) „Wissenstransfer", Infopaket $360^{\circ}$ Onkol., Nr. Ausgabe 8. https://www.krebsgesellschaft. de/deutsche-krebsgesellschaft-wtrl/deutschekrebsgesellschaft/gesundheitspolitik/id-360onkologie-ausgaben-2019.html?file=files/ $\mathrm{dkg} /$ deutsche-krebsgesellschaft/content/pdf/ Gesundheitspolitik/360\%20Grad\%20Printaus gaben/2019/08_2019_Printnewsletter.pdf\&cid $=61432$. Zugegriffen:7. Juni 2021

\section{Europe's Beating Cancer Plan. Let's strive for more}

At the level of the European Union (EU), the fight against cancer is currently high on the list of priorities in the area of health. In this context, the European Commission presented Europe's Beating Cancer Plan (EBCP) in February 2021. This article gives an insight into the background, structure, and implementation of the EBCP. Herein, selected actions of the EBCP are described based on the four pillars of prevention, early detection, diagnosis, and treatment, as well as regards improving the quality of life of cancer patients and survivors. Although much is still in the early stages, the article also provides a preview of the first steering and monitoring initiatives that the EU commission has undertaken to implement the plan. The focus is on interdisciplinary cooperation between research and care-as laid out in the Cancer Plan and as is increasingly taking place in Germany.

\section{Keywords}

Health policy · European Policy · Early detection of cancer · Quality of life · Quality of care 